\title{
Assessment of Cytokine mRNA Expression Profiles in Tumor Microenvironment and Peripheral Blood Mononuclear Cells of Patients with High-grade Serous Carcinoma of the Ovary
}

\section{Pernilla Israelsson ${ }^{1,2}$, Alireza Labani-Motlagh ${ }^{1}$, Ivan Nagaev ${ }^{1}$, Eva Dehlin ${ }^{1}$, Olga Nagaeva ${ }^{1}$, Eva Lundin ${ }^{3}$, Ulrika Ottander ${ }^{2}$ and Lucia} Mincheva-Nilsson ${ }^{1 *}$

${ }^{1}$ Departments of Clinical Microbiology/Clinical Immunology, Umeå University, Umeå, Sweden

${ }^{2}$ Departments of Obstetrics and Gynecology, Umeå University, Umeå, Sweden

${ }^{3}$ Departments of Medical Biosciences/Pathology, Umeå University, Umeå, Sweden

\begin{abstract}
Objective: Tumor establishment, metastatic spreading and poor survival in ovarian cancer is strongly associated with progressive derangement of the patient's immune system. Accumulating evidence suggests that immune impairment is influenced by the production and presence of cytokines in the tumor microenvironment.

Methods: Cytokine mRNA profiles in tumor tissue and peripheral blood mononuclear cells (PBMC) were analyzed in patients with high grade serous carcinoma (HGSC) of the ovary and compared it to patients with benign ovarian conditions and controls with normal ovaries. Cytokine assessment was done by real-time quantitative RT-PCR and specific primers and probes for 12 cytokines-IFN-y, IL-1 $\beta$, IL-2, IL-4, IL-6, IL-8, IL-10, IL-15, TNF- $\alpha$, TNF- $\beta / L T A$, TGF- $\beta 1$, and GM-CSF chosen to distinguish between cytotoxic Th1, humoral Th2, regulatory Th3/Tr1 and inflammatory responses.

Results: The cytokine mRNA response in the HGSC patients was significantly up regulated compared to patients with benign ovarian conditions and normal ovary controls confirming the immunogenicity of HGSC and implying immune recognition and reaction locally in the tumor microenvironment and systemically in the peripheral blood.There was an up-regulation of inflammatory and inhibitory cytokine mRNA promoting tumor progression, T-regulatory cell priming and T-regulatory cell-mediated immune suppression. In contrast, there was an inability to mount the crucially important IFN gamma response needed for upregulation of the cytotoxic anti-tumor response in the local microenvironment. In addition, systemic IL-4- mediated Th2 response prevailed in the peripheral blood deviating the systemic defense towards humoral immunity.
\end{abstract}

Conclusions: Taken together, these results suggest local and systemic cytokine cooperation promoting tumor survival, progression and immune escape. Our study confirms and extends previous investigations and contributes to the evaluation of potential cytokine candidates for diagnostic cytokine mRNA profiles and for future therapeutic interventions based on cytokine inhibition.

Keywords: Cytokines; High-grade serous ovarian carcinoma (HGSC); EOC; Tumor microenvironment; Tumor inflammation; Immune suppression

\section{Introduction}

Several reports indicate that tumors develop mechanisms to subvert the immune responses and suppress immune surveillance [1]. Previous studies have shown that these mechanisms down regulate the anti-tumor immunity of the patient. The tumor can directly inhibit the immune cells' function by deviation of their responses, down regulation of cellular receptors or suppression of their mechanisms of action [13]. Several of these mechanisms are promoted by immunosuppressive cytokines, chemokines and growth factors that are raised and operate in the tumor microenvironment $[3,4]$. Ovarian cancer is the most lethal of all gynecological malignancies [5]. It is heterogeneous in nature and classified based on histological type where the epithelial ovarian carcinoma (EOC) is the most common and comprises about $90 \%$. EOC can be subdivided into high-grade serous carcinoma (HGSC), endometroid, clear-cell, mucinous and low-grade serous carcinoma. The high-grade serous type is the most abundant and malignant [6]. A new classification was presented by Shih and Kurman subdividing the tumors according to tumorigenesis and genetic features into type I and type II [7]. Type I tumors are slow growing and genetically stable. Type II tumors are more aggressive, present themselves at a late stage and are genetically unstable. Since ovarian cancer has diffuse symptoms and there is no specific diagnostic marker(s), most women are diagnosed with metastasized disease (FIGO stages III and IV) [2]. Cytokines, modulating immune responses, may play a significant role in the establishment and progression of ovarian cancer [8]. Cytokines are cell-signaling proteins/peptides for intercellular communication, secreted by a variety of cells. Different cytokine profiles, designated Th1, Th2, Th3, Tr1 and Th17, are associated with the ability to mediate and regulate immunity and inflammation, promote or halt cell growth and movement or immune responses. Thus, a cytokine profile dominated by IFN- $\gamma$ and interleukin (IL)-15 (Th1) promotes cytotoxicity, a cytokine

*Corresponding author: Lucia Mincheva-Nilsson, Departments of Clinical Microbiology/Clinical Immunology, Umeå University, Umeå, Sweden, Tel: 46907852237; E-mail: lucia.mincheva-nilsson@umu.se

Received May 02, 2017; Accepted May 19, 2017; Published May 22, 2017

Citation: Israelsson P, Labani-Motlagh A, Nagaev I, Dehlin E, Nagaeva O et al. (2017) Assessment of Cytokine mRNA Expression Profiles in Tumor Microenvironment and Peripheral Blood Mononuclear Cells of Patients with High-grade Serous Carcinoma of the Ovary. J Cancer Sci Ther 9:422-429. doi: 10.4172/1948-5956.1000453

Copyright: (c) 2017 Israelsson P, et al. This is an open-access article distributed under the terms of the Creative Commons Attribution License, which permits unrestricted use, distribution, and reproduction in any medium, provided the original author and source are credited. 
Citation: Israelsson P, Labani-Motlagh A, Nagaev I, Dehlin E, Nagaeva O, et al. (2017) Assessment of Cytokine mRNA Expression Profiles in Tumor Microenvironment and Peripheral Blood Mononuclear Cells of Patients with High-grade Serous Carcinoma of the Ovary. J Cancer Sci Ther 9: 422-429. doi: 10.4172/1948-5956.1000453

profile dominated by IL-4 (Th2) promotes humoral immunity, while IL-17 (Th17), IL-1 $\beta$, IL-6, IL-8, tumor necrosis factor (TNF)- $\alpha$ and TNF- $\beta$ /LTA promote inflammation, and TGF- $\beta$ and IL-10 (Th3, $\mathrm{Tr} 1)$ promote $\mathrm{T}$ regulatory cell-induced immunosuppression. These cytokines can be expressed in small amounts in the normal ovaries as well [8]. Previous assessments of protein concentration of cytokines in serum and ascites of EOC patients and in supernatants of EOC cell lines have shown that there is a shift towards immunoinhibitory cytokines [8-13]. Additionally, in earlier reports from the nineties, attempts were made to assess mRNA expression for a limited number of individual cytokines in tumor tissue using Northern blot or semi-quantitative RTPCR [14-17].

Here, we quantified the mRNA transcription of a broad number of cytokines in paired samples of tumor tissue and peripheral blood mononuclear cells (PBMC) from HGSC, benign ovarian conditions and normal controls by real-time RT-qPCR. The cytokine primers and probes used distinguish between the main cytokine mRNA patterns, i.e. Th1-, Th2-, Th3/Tr1-and inflammatory pattern, known to be the key cytokines that control cytotoxic-, humoral-, regulatory- and inflammatory responses respectively. Our goal was to elucidate the local cytokine mRNA production in the tumor tissue and to compare it to the systemic cytokine profile measured in PBMC of the same patients as well as to compare it to benign ovarian conditions and normal ovaries aiming to reveal differences in the systemic and local modulation of the patient's immune response to HGSC.

\section{Materials and Methods}

\section{Ethical statement}

This investigation was approved by the Human Ethics Committee of the Medical Faculty, Umeå University (dnr 09-108M). All patients and controls donated samples after informed consent.

\section{Patient samples}

Tissue samples from patients with HGSC/EOC type II of the ovary, benign ovarian conditions, normal ovaries and corresponding peripheral blood samples were collected between 2005 and 2015 at surgery from 22 women with HGSC, 19 women with benign ovarian conditions and 8 with normal ovaries. Information about the patients and the histopathologic diagnosis according to the World Health Organization Classification [18], was extracted from their medical records.

\section{Preparation of tissue and blood samples}

Tissue samples, snap-frozen in liquid nitrogen and stored at $-150^{\circ} \mathrm{C}$, and corresponding Buffy coats from the patients with HGSC, benign ovarian conditions and normal ovaries were retrieved from the Ovarian Cancer Biobank at Norrland's University Hospital. PBMC were isolated from Buffy coats by Lymphoprep (Nycomed) gradient centrifugation as previously described $[19,20]$. The collected interphase containing lymphocytes and macrophages was washed, counted, frozen and kept at $-80^{\circ} \mathrm{C}$ until use.

\section{Total RNA extraction and real-time quantitative reverse transcription-polymerase chain reaction (RT-qPCR)}

Cytokine gene expression analysis was performed by real time RT-qPCR following the MIQE requirements [21]. A summary of the positive samples from the total number of samples for the individual cytokines analyses are given in Table 1 .

RNA extraction: About $0.5 \mathrm{mg}$ of frozen ovarian tissue/sample was sliced into $25 \mu \mathrm{m}$ slices in a cryostatic microtome, dissolved in $350 \mu \mathrm{l}$ of lysis buffer and total RNA was extracted using RNeasy Mini Kit (Qiagen, Hilden, Germany). PBMC from Buffy coats were thawed by layering $200 \mu \mathrm{l}$ RNA later stabilization solution (AM7020, ThermoFisher Scientific inc., USA) mixed by pipetting and transferred to a tube with TRIzol Reagent (ThermoFisher Scientific). Total RNA was isolated and cleaned up with RNeasy Mini kit. RNA yield (on average $3961 \mathrm{ng}$ in total volume of $50 \mu \mathrm{l}$ ) and purity (average $\mathrm{A}_{260} / \mathrm{A}_{280}=1.7$ ) were assessed by spectrophotometry (NanoDrop, ThermoScientific).

Reverse transcription: For each sample $400 \mathrm{ng}$ total RNA in a reaction volume of $20 \mu \mathrm{l}$ was transcribed into cDNA by random hexamers, MuLV reverse transcriptase and dNTP mix using highcapacity cDNA reverse Transcription Kit (ThermoFisher) according to the manufacturer's description. Thereafter, $60 \mu \mathrm{l}$ sterile miliQ water was added to each sample to adjust the cDNA concentration to $5 \mathrm{ng} /$ $\mu$ total RNA.

\begin{tabular}{|c|c|c|c|c|c|c|c|c|c|}
\hline \multirow{2}{*}{\begin{tabular}{|l|} 
Group \\
Samples
\end{tabular}} & \multicolumn{2}{|c|}{ EOC } & \multicolumn{2}{|c|}{ Benign } & \multicolumn{2}{|c|}{ Normal } & \multicolumn{2}{|c|}{ Assay controls } & \multirow{3}{*}{$\begin{array}{c}\text { Assay ID } \\
\text { (ThermoFisher Scientific) }\end{array}$} \\
\hline & ovary & blood & ovary & blood & ovary & blood & sPBMC & 18S rRNA & \\
\hline Total number & 22 & 14 & 18 & 18 & 8 & 7 & 1 & 88 & \\
\hline Gene & \multicolumn{6}{|c|}{ Number of positive samples } & \multicolumn{2}{|c|}{ Ct value* } & \\
\hline IL1 $\beta$ & 22 & 14 & 15 & 13 & 8 & 4 & 21.7 & $16.0 \pm 2.03$ & Hs01555410_m1 \\
\hline IL2 & 14 & 9 & 10 & 11 & 4 & 4 & 26.7 & $14.4 \pm 1.81$ & Hs00174114_m1 \\
\hline IL4 & 8 & 12 & 7 & 10 & 3 & 3 & 35.5 & $15.3 \pm 1.91$ & Hs00174122_m1 \\
\hline IL6 & 22 & 13 & 16 & 8 & 8 & 4 & 26.4 & $15.1 \pm 1.81$ & Hs00985639_m1 \\
\hline IL8 & 22 & 12 & 14 & 18 & 8 & 7 & 20.7 & $15.9 \pm 2.10$ & Hs00174103_m1 \\
\hline IL10 & 21 & 10 & 15 & 11 & 7 & 4 & 28.5 & $14.2 \pm 1.91$ & Hs00961622_m1 \\
\hline IL15 & 22 & 14 & 15 & 13 & 7 & 6 & 32.3 & $14.7 \pm 1.76$ & Hs01003716_m1 \\
\hline TNFa & 22 & 14 & 13 & 14 & 8 & 5 & 25.6 & $16.3 \pm 2.08$ & Hs00174128_m1 \\
\hline TNF $\beta / L T A$ & 20 & 12 & 13 & 13 & 6 & 4 & 26.6 & $15.8 \pm 1.97$ & Hs04188773_g1 \\
\hline TGF $\beta 1$ & 21 & 14 & 13 & 16 & 7 & 5 & 24.9 & $15.4 \pm 1.94$ & Hs99999918_m1 \\
\hline IFNy & 21 & 11 & 12 & 11 & 6 & 4 & 25.2 & $15.7 \pm 1.92$ & Hs00989291_m1 \\
\hline GM-CSF & 17 & 7 & 5 & 10 & 5 & 5 & 28.2 & $15.2 \pm 1.88$ & Hs00929873_m1 \\
\hline
\end{tabular}

Table 1: Number of positive samples, average Ct-values for assay-and house-keeping gene controls and gene assay ID. 
Citation: Israelsson P, Labani-Motlagh A, Nagaev I, Dehlin E, Nagaeva O, et al. (2017) Assessment of Cytokine mRNA Expression Profiles in Tumor Microenvironment and Peripheral Blood Mononuclear Cells of Patients with High-grade Serous Carcinoma of the Ovary. J Cancer Sci Ther 9: 422-429. doi: 10.4172/1948-5956.1000453

Real-time quantitative PCR amplification: Multiplexed qPCR tests were performed detecting the target gene and 18S rRNA as endogenous control on an ABI PRISM 7900HT Sequence Detection Instrument (Applied Biosystems). One $\mu \mathrm{l} \mathrm{cDNA} /$ well in $20 \mu \mathrm{l}$ reaction volume was used in all tests and run for 40 cycles with factory default settings for TaqMan Gene Expression Master Mix (\#4369016) and TaqMan $^{\circ}$ FAM/MGB probe Assays (all purchased from Thermo Fisher). The following cytokines were assessed: IFN- $\gamma$, IL- $1 \beta$, IL-2, IL4, IL-6, IL-8, IL-10, IL-15, TNF- $\alpha$, TNF- $\beta / L T A$, TGF- $\beta 1$, and GM-CSF. The catalogue numbers are given in Table 1 . The constancy of $\mathrm{Ct}$ values for the endogenous, house-keeping gene $18 \mathrm{~S}$ rRNA is shown in Table 1. The Ct values were similar between samples (standard deviation on average was 1.5) with a normal variation between different gene assays but very stable during the whole study. Cytokine mRNA amplifications of PMA-ionomycin stimulated PBMCs from healthy donors were used as positive assay controls and template omission as a negative control. The obtained cytokine mRNA profiles of tissue and blood were compared between patients with HGSC, patients with benign ovarian conditions and controls with normal ovaries. Data was analyzed with AB 7900 Sequence Detection Software version 2.4.

\section{Statistical Analysis}

Comparative $\mathrm{Ct}(\Delta \Delta \mathrm{Ct})$ method was applied for computing relative quantities (RQ) and an average RQ (aRQ) was calculated for each study group. Every individual fold difference value is a result of division of a test group aRQ by a reference group aRQ, thus standard error and standard deviation is not applicable for calculation and presentation of the results. Statistical tests were used to estimate the variance for each group. We used Student's T test to evaluate statistical significance. P-values $\leq 0.05$ were considered significant.

\section{Results}

\section{Patients}

Twenty-two patients with HGSC/serous EOC type II diagnosis, aged from 45 to 77 years, participated in the study. The characteristics of the patients are presented in Tables 2,3 and 4. The differentiation grade was low, except for 3 patients where it was low to moderate and 1 where it was undifferentiated (Table 2). Six of the patients had received neoadjuvant preoperative chemotherapy. Standard cytostatic treatment according to guidelines [22] was given for 2-4 cycles, 3 weeks apart, surgery following within 6 weeks of the last cycle. The cytokine mRNA profiles of the patients treated with cytostatic drugs $(n=6)$ are presented separately. Of the remaining 16 patients with HGSC, 14 had both tissue and blood samples. Twenty patients with benign ovarian conditions were included in the benign group, 18 of those had corresponding ovarian tissue- and peripheral blood samples. Their individual diagnoses are presented in Table 3. The normal control group, presented in Table 4, consisted of 8 women with normal healthy ovaries. All patients were matched referring to age and body mass index (BMI). The peripheral blood samples were taken on the day of operation. There were blood samples available from 14 HGSC/EOC patients, 18 patients with benign ovarian conditions and from 7 with normal ovarian tissue, annotated in the tables as paired tissue- and peripheral blood samples.

The cytokine mRNA profiles in paired HGSC tissue and PBMC samples revealed a statistically significant mobilization of inflammatory and T-regulatory responses combined with an enhanced systemic humoral response and a failure to mount a local cytotoxic response

The cytokine mRNA profiles in tissue- and blood samples from

\begin{tabular}{|c|c|c|c|c|c|c|}
\hline Patient $(n=22)$ & Stage (FIGO) & Grade of differentiation & Age & $\mathrm{BMI}^{\mathrm{a}}$ & Paired samples tissue and blood ${ }^{b}$ & Preop chemoc \\
\hline 1 & II & low & 70 & 27 & - & - \\
\hline 2 & IV & low & 56 & 20,9 & - & - \\
\hline 3 & IV & low & 52 & 23,4 & - & - \\
\hline 4 & II & low & 45 & 22,3 & - & - \\
\hline 5 & IV & low & 54 & 32,3 & + & + \\
\hline 6 & III & low & 55 & 21,3 & + & - \\
\hline 7 & III & low & 63 & 28,6 & + & - \\
\hline 8 & III & low & 56 & 30,6 & + & + \\
\hline 9 & III & low & 63 & 29,3 & + & + \\
\hline 10 & III & low & 76 & 34,1 & + & - \\
\hline 11 & 1 & low & 77 & 24,5 & + & - \\
\hline 12 & III & low & 54 & 29,4 & + & - \\
\hline 13 & III & low & 56 & 32,5 & + & - \\
\hline 14 & IV & low & 56 & 21,1 & - & - \\
\hline 15 & III & low & 67 & 22,5 & + & - \\
\hline 16 & III & low & 68 & 21,6 & + & - \\
\hline 17 & IV & low & 70 & 23,5 & + & - \\
\hline 18 & III & low & 51 & 22,5 & - & - \\
\hline 19 & IV & low/moderate & 51 & 21,9 & - & + \\
\hline 20 & 1 & low/moderate & 75 & 29,6 & + & - \\
\hline 21 & III & low/moderate & 49 & 28,4 & + & + \\
\hline 22 & III & undifferentiated & 54 & 23,2 & - & - \\
\hline \multicolumn{3}{|l|}{ Mean \pm SD } & $60,2 \pm 9$ & $26,4 \pm 5$ & & \\
\hline \multicolumn{7}{|c|}{$\begin{array}{l}\text { body mass index } \\
\text { b + paired samples of blood and tissue available } \\
\text { - no blood sample } \\
\text { c + received preoperative chemotherapy } \\
\text { - no preoperative chemotherapy }\end{array}$} \\
\hline
\end{tabular}

Table 2: Characteristics of patients with HGSC of the ovary. 
Citation: Israelsson P, Labani-Motlagh A, Nagaev I, Dehlin E, Nagaeva O, et al. (2017) Assessment of Cytokine mRNA Expression Profiles in Tumor Microenvironment and Peripheral Blood Mononuclear Cells of Patients with High-grade Serous Carcinoma of the Ovary. J Cancer Sci Ther 9: 422-429. doi: 10.4172/1948-5956.1000453

\begin{tabular}{|c|c|c|c|c|}
\hline Patient $(n=20)$ & Diagnosis & Age & $\mathrm{BMI}^{\mathrm{a}}$ & Paired samples tissue and blood \\
\hline 1 & Serous cysts ${ }^{c}$ & 71 & 22 & + \\
\hline 2 & Serous cysts & 66 & 26,4 & + \\
\hline 3 & Serous cyst & 18 & 20,6 & + \\
\hline 4 & Serous cyst & 65 & 25,6 & + \\
\hline 5 & Serous cystadenoma & 74 & 25,3 & + \\
\hline 6 & Serous cystadenoma & 59 & 24,8 & + \\
\hline 7 & Serous cystadenoma & 74 & 17,4 & + \\
\hline 8 & Serous cystadenoma & 68 & 30,2 & + \\
\hline 9 & Serous cystadenoma and torsion & 59 & 22,4 & + \\
\hline 10 & Serous cystadenofibroma & 57 & 26,3 & + \\
\hline 11 & Serous cystadenofribroma & 24 & 46,1 & + \\
\hline 12 & Serous cystadenofibroma & 53 & 25,7 & + \\
\hline 13 & Mucinous cystadenoma & 45 & 21,8 & - \\
\hline 14 & Mucinous cystadenoma & 66 & 34,4 & - \\
\hline 15 & Mucinous cystadenoma & 72 & 31,1 & + \\
\hline 16 & Mucinous cystadenoma & 68 & 24,4 & + \\
\hline 17 & Cyst $^{\mathrm{c}}$, unspecific & 71 & 22,7 & + \\
\hline 18 & Cysts $^{\mathrm{c}}$, unspecific & 68 & 25,6 & + \\
\hline 19 & Inclusion cysts ${ }^{c}$ & 52 & 33,2 & + \\
\hline 20 & Torsion & 48 & 24,5 & + \\
\hline \multicolumn{2}{|l|}{ Mean \pm SD } & $58,9 \pm 15,6$ & $26,5 \pm 6,2$ & \\
\hline \multicolumn{5}{|c|}{$\begin{array}{l}\text { a body mass index } \\
\text { b }+ \text { paired samples of blood and tissue available } \\
- \text { no blood sample } \\
\text { c } \geq 8 \mathrm{~mm} \text { in size }\end{array}$} \\
\hline
\end{tabular}

Table 3: Characteristics of patients with benign conditions.

\begin{tabular}{|c|c|c|c|c|}
\hline Patient $(n=8)$ & Diagnosis & Age & $\mathrm{BMI}^{\mathrm{a}}$ & Paired samples tissue and blood ${ }^{b}$ \\
\hline 1 & Normal tissue & 41 & 21.6 & + \\
\hline 2 & Normal tissue & 75 & 21.8 & + \\
\hline 3 & Normal tissue & 57 & 27.1 & - \\
\hline 4 & Serous inclusion cysts ${ }^{b}$ & 63 & 19.4 & + \\
\hline 5 & Serous inclusion cysts & 54 & 17 & + \\
\hline 6 & Serous inclusion cysts & 73 & 19.9 & + \\
\hline 7 & Inclusion cyst ${ }^{c}$ & 67 & 33.7 & + \\
\hline 8 & Inclusion cysts ${ }^{c}$ & 71 & 32.9 & + \\
\hline \multicolumn{2}{|c|}{ Mean $\pm S D$} & $63.9 \pm 11$ & $24.2 \pm 6.3$ & \\
\hline
\end{tabular}

Table 4: Characteristics of patients with normal ovarian tissue.

cytostatic drug-treated and untreated HGSC patients are presented in Figure $1 \mathrm{~A}$ and $1 \mathrm{~B}$. As can be seen there was an enhanced cytokine mRNA expression in the untreated EOC patients (Figure 1A) compared to normal controls. Most prominent and significant mRNA upregulation in both tumor tissue and the corresponding peripheral blood was found for IL-1 $\beta$, IL-6, IL-8, TNF $\alpha$ and TNF $\beta /$ LTA i.e cytokines mediating inflammatory response. The upregulation was more prominent in the tumor tissue compared to peripheral blood (Figure 1A). One of the cytokines, IL-8 was highly upregulated in the tumor tissue while in the peripheral blood the expression was somewhat lower or unchanged (Figure 1A). Both TNF $\alpha$ and TNF $\beta /$ LTA, the latter an inflammatory cytokine secreted only by immune cells, were significantly upregulated (Figure 1A). The second important observation was that, in the tumor tissue, there was a highly significant upregulation of the T regulatory responses measured by TGF- $\beta 1$ mRNA (innate- and/or Th3 adaptive T-regulatory cells) and IL-10 mRNA (adaptive Tr1 regulatory cells). The mRNA upregulation in the peripheral blood reached higher significance compared to tumor tissue in the HGSC patients compared to normal controls (Figure 1A). In contrast, IFN $\gamma$ was unchanged or downregulated without reaching a statistical significance. Messenger RNA for IL-15, another Th1-oriented cytokine, remained also statistically unchanged (Figure 1A). IL-4, the index cytokine of humoral immune responses was differentially expressed in tumor tissue and peripheral blood. Although not reaching a statistical significance, IL-4 was downregulated in the tumor tissue but significantly upregulated in the peripheral blood (Figure 1A). Interleukin-2 mRNA was enhanced in the tumor microenvironment compared to PBMC (Figure 1A). In Figure $1 \mathrm{~B}$ the cytokine mRNA profile of the 6 patients that received preoperative chemotherapy is presented. As can be seen the overall cytokine mRNA expression was highly depressed compared to the normal control group although statistical significance could not be achieved due to the small number of patients.

The cytokine mRNA expression in paired tissue and PBMC samples of benign ovarian conditions is generally much lower than in HGSC and comparable to the mRNA expression in normal ovarian tissue and PBMC

In Figure 2 the relative cytokine mRNA expression in benign 
Citation: Israelsson P, Labani-Motlagh A, Nagaev I, Dehlin E, Nagaeva O, et al. (2017) Assessment of Cytokine mRNA Expression Profiles in Tumor Microenvironment and Peripheral Blood Mononuclear Cells of Patients with High-grade Serous Carcinoma of the Ovary. J Cancer Sci Ther 9: 422-429. doi: 10.4172/1948-5956.1000453

ovarian conditions is compared to the normal control group. The first observation is that generally, the cytokine mRNA expression in benign conditions is much lower compared to HGSC/ EOC. As seen in Figure $1 \mathrm{~B}$ and Figure 2 the fold change in mRNA expression was up to 16 to 32 times higher and statistically significant in HGSC/EOC patients, while the fold change in patients with benign ovarian conditions was at the most 2 to 4 times higher and did not reach statistically significant levels. Furthermore, mRNA expression for several inflammatory cytokines was downregulated in the peripheral blood suggesting that the benign ovarian conditions in this patient group did not evoke a systemic cytokine response.

Cytokine mRNA profiles in HGSC compared to benign ovarian conditions reveal a statistically significant enhancement of inflammatory and T-regulatory responses, a failure to mobilize a local Th1-primed cytotoxic anti-tumor response and a systemic Th2-primed humoral response

In Figure 3 a comparison between the cytokine mRNA profile in tumor tissue and PBMC in HGSC patients and that of tissue and PBMC in patients with benign ovarian conditions is presented. The relative mRNA expression in HGSC is shown as a fold change compared to the relative mRNA expression in tissue and PBMC of benign ovarian conditions shown in the Figure as $=1$. In general, there was a statistically significant increase in the cytokine mRNA expression in ovarian cancer tissue and peripheral blood compared to the ovarian tissue and blood from patients with benign conditions. Two significantly enhanced cytokine responses, an inflammatory and a regulatory response, prevailed both in the tumor microenvironment and the peripheral blood Figure 3. In contrast, there was a failure in mounting a Th1 response measured as IFN $\gamma$ mRNA response which
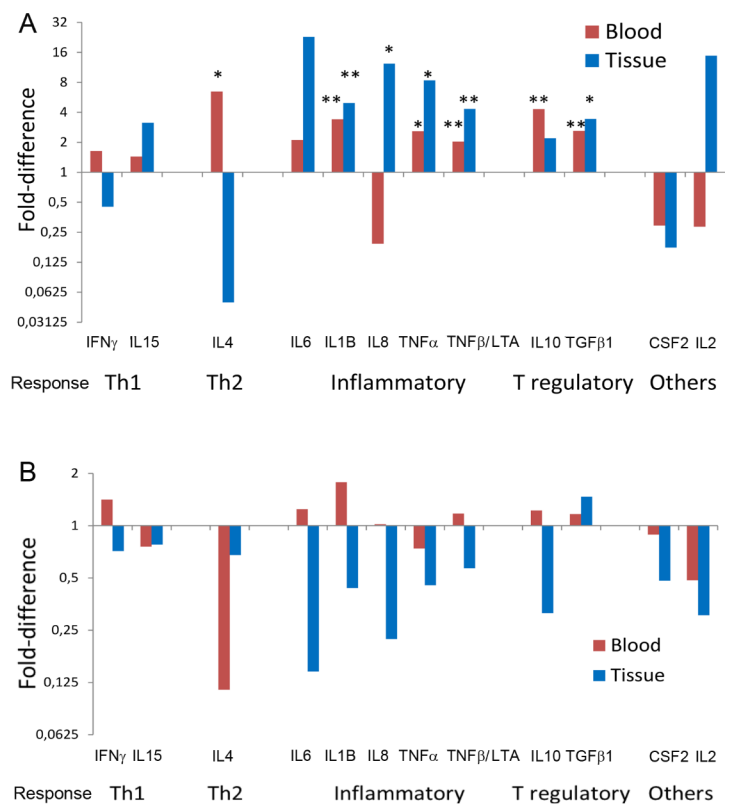

Figure 1: Relative cytokine mRNA expression in non-treated (A) and preoperatively cytostatic drug-treated (B) patients with high-grade serous carcinoma of the ovary (HGSC). Results are presented as a fold difference between average of mRNA expression level in paired tissue- and PBMC samples from HGSC patients and a control group $(=1)$ with healthy ovaries and therefore no standard deviation is given. Statistical significance is calculated with Student's T test. ${ }^{*}=p<0.05 ;{ }^{* *}=p<0.001$.

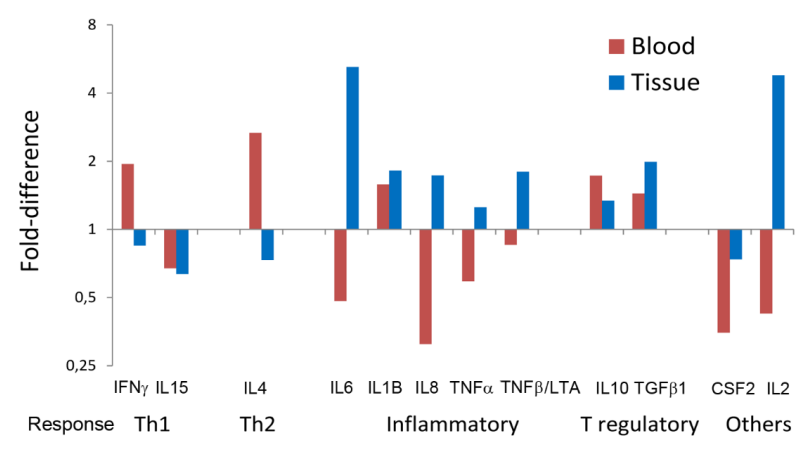

Figure 2: Relative cytokine mRNA expression in patients with benign ovarian conditions. Fold difference in cytokine expression levels between paired tissue- and PBMC samples from patients with benign ovarian conditions and control group $(=1)$ with healthy ovaries.

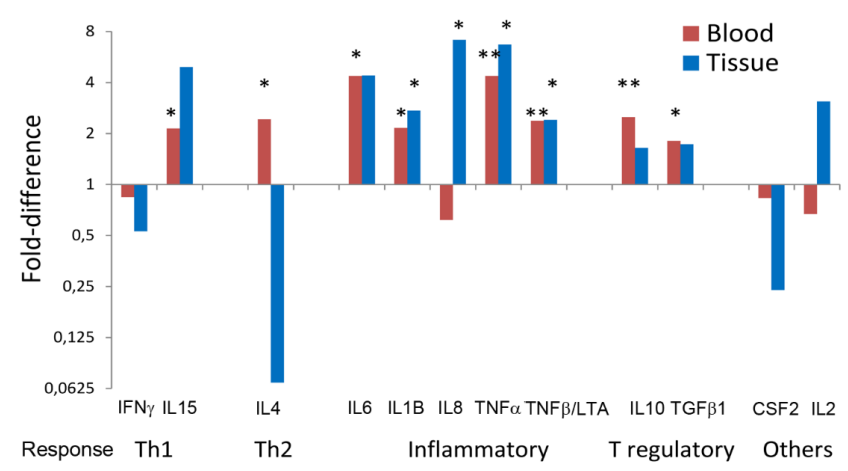

Figure 3: Relative cytokine mRNA expression in patients with high-grade serous carcinoma of the ovary (HGSC) compared to the mRNA expression in patients with benign ovarian conditions. Fold difference in cytokine expression levels between paired tissue- and PBMC samples from HGSC patients and patients with benign ovarian conditions $(=1) .{ }^{*}=p<0.05 ;{ }^{* *}=p<0.001$.

was slightly downregulated/unchanged in tissue and blood of the HGSC patients. There was a significantly enhanced IL-15 mRNA response in the peripheral blood of the HGSC patients suggesting a systemic Th1 response. Interleukin-4 was downregulated in the tumor tissue but significantly enhanced in PBMC.

\section{Discussion}

In this investigation, we assessed the mRNA profile for 12 cytokines in ovarian tumor tissue samples and corresponding PBMC from HGSC patients using real-time RT-qPCR and compared it to corresponding analyses in benign ovarian conditions and normal ovaries. Our results can be summarized as follows: 1) The cytokine mRNA expression in tumor tissue and corresponding PBMC of HGSC patients was significantly upregulated compared to patients with benign ovarian conditions and normal ovaries confirming the immunogenicity of EOC and implying an immune recognition and reaction locally in the tumor microenvironment and systemically in peripheral blood. 2) The significantly enhanced cytokine mRNA expression in HGSC patients was oriented towards inflammation and innate and adaptive $\mathrm{T}$ regulatory cells, i.e. responses suppressing anti-tumor immunity and promoting tumor growth and metastasis. 3) In contrast, the IFN $\gamma$ response that would promote cytotoxicity needed for tumor cell elimination was unchanged or downregulated. 4) The absence of Th1 
oriented response was combined with a deviation towards systemic humoral Th2 response. 5) Additionally, mRNA for IL-2, a cytokine promoting clonal expansion of immune cells and GM-CSF, a cytokine shown to be associated with EOC prognosis, were differentially expressed in HGSC patients suggesting an unsuccessful attempt to activate anti-tumor immunity and promoting $\mathrm{T}$ regulatory cell responses instead. 6) And finally, the cytokine mRNA expression in the 6 HGSC patients that received pre-operative cytostatic treatment was generally and highly depressed showing that preoperative cytostatic therapy knocks down the immune system preventing cytokine mRNA expression thus abrogating activation of any immune response.

HGSC/EOC is known to harbor tumor infiltrating lymphocytes (TILs) in various amounts that could be correlated to the overall patient survival (reviewed in 1). Depending on the subtypes of TILs, they may play opposite roles in the disease outcome. A high frequency of cytotoxic $\mathrm{CD}^{+} \mathrm{T}$-cells, NK cells and $\mathrm{V} \gamma 9 \mathrm{~V} \delta 2 \gamma \delta \mathrm{T}$ cells in the TIL cell population was correlated with better survival rates [23-25] whereas dominance of Tregs among TILs created an immunosuppressive microenvironment helping the tumor escape immune attack [26]. We found differential cytokine mRNA expression in the tumor microenvironment and PBMC of HGSC patients, patients with benign ovarian conditions and the normal control group. Firstly, the HGSC patients could not mount an adequate mRNA expression of the Th1 index cytokine INF $\gamma$ which promotes T-cell mediated cytotoxicity crucially important in anti-cancer defense. The downregulation of IFN $\gamma$ expression in the tumor microenvironment was accompanied by enhancement of IL-15 mRNA signals. IL-15 has been previously shown to activate binding of STAT1-5 to regulatory sites of the IFN $\gamma$ gene thus participating in IFN $\gamma$ up-regulation [27]. So the observed increase in IL-15 mRNA might be a compensatory but unsuccessful attempt to up-regulate the IFN $\gamma$ expression. Secondly, in line with the dysregulated Th1 response there was an up-regulated IL-4 mRNA response in PBMC suggesting a Th2 deviation of the systemic immune response that further disrupts Th1-mediated cytotoxic function thus promoting immune escape of the tumor. Thirdly, both in the tumor microenvironment and in the peripheral blood prevailed a constitutive and statistically significant mRNA up-regulation of the immunosuppressive cytokines TGF $\beta$, IL-10, and IL-2, all three being cytokines that are instrumental for the priming, clonal expansion and immunosuppressive function of both innate and adaptive $\mathrm{T}$ regulatory cells [28]. The up-regulation of these cytokines is consistent with the assumption that TILs of T regulatory phenotype are present in the analyzed tumor tissue samples. Accumulation of Treg in most tumors, including epithelial ovarian tumors, is a well-documented fact. T regulatory cells of both innate and adaptive type have emerged as "cancer's best friends" in that they act as major immune suppressors of the immune response and thus largely contribute to the compromised status of the immune system of cancer patients [29]. A specific recruitment of $\mathrm{CD} 4^{+} \mathrm{CD} 25^{+} \mathrm{FoxP} 3^{+}$ Treg cells, tolerogenic dendritic cells, tumor-associated macrophages and myeloid-derived suppressor cells takes place in the tumor microenvironment of HGSC/EOC [30,31], all of these cells being involved in the immune escape of EOC and coinciding with rapid tumor progression and poor survival [32]. In addition to recruitment, there is local Treg expansion and de novo conversion of FoxP3- naïve $T$ cells into adaptive Tregs in the tumor microenvironment under the influence of TGF $\beta$, IL-10 and IL-2 $[29,33,34]$. Thus, TGF $\beta$, IL-10 and IL-2 mRNA profile in our analyses suggests Tregs in the TIL population. In addition to the negative influence of Treg cells in tumors, the role of the newly discovered innate lymphoid cells (ILC) should be of interest to study. ILC in cancer have been little studied so far, however, recent reports suggest a role for ILC subclasses both in the promotion and maintenance but also in the elimination of tumors at various anatomical sites (reviewed in 35). Recent observations indicated that epigenetic mechanisms might be involved in the regulation of ILC and $\mathrm{T}$ cells via the enzyme histone lysine methyltransferase G9a. This enzyme regulates ILC2 development and function and, in addition, the T-cell differentiation during inflammation as shown in a mouse model [35-38]. In a G9a double knock out murine model the T cell response was deviated towards inflammation and $\mathrm{T}$ regulatory-cell priming, i.e. tumor promoting events [38]. Thus, aberrant G9a expression might affect the immune cell homeostasis in malignant human disorders. These findings, and the participation of G2a and its partner enzyme GLP in maintenance of DNA methylation $[39,40]$ suggest that G9a might serve as an immunological check point and also as an epigenetic regulator and therefore be a good candidate for intervention in future anti-cancer therapy.

A constitutive finding in our analyses was a high and statistically significant up-regulation of the inflammatory response in HGSC locally in the tumor microenvironment and systemically in the PBMC as measured by cytokine mRNA expression of IL- $1 \beta$, IL- 6 , IL-8, TNF $\alpha$ and TNF $\beta /$ LTA. In contrast, benign ovarian conditions expressed lower amount of inflammatory cytokine mRNA comparable to normal ovarian tissue and PBMC of the normal control patients. The local inflammatory mRNA up-regulation in HGSC tumor tissue was more prominent than that in the corresponding PBMC of paired samples suggesting an enrichment of inflammatory cytokine mRNA signals in the local tumor micromillieau. Tumor-associated chronic inflammation is not specific for ovarian cancer but is a constitutive feature in cancer per se $[41,42]$. Compelling evidence imply that the inflammatory cytokine response in ovarian cancer promotes tumorigenesis and influences the clinical disease status, progress and prognosis $[43,44]$. Most of the inflammatory cytokines included in our analysis, such as TNF- $\alpha$, IL- $1 \beta$, IL- 6 , and IL- 8 can be produced both by the ovarian tumor itself, supporting stromal cells and/or by activated tumor- infiltrating immune cells and all of these cell types participate in the molding of inflammation in the local tumor microenvironment [42-44]. We have analyzed the general cytokine milieu in the tumor microenvironment without dividing the cytokine response into tumor cell-, stromal cell- or immune-cell associated. The reason for that is that we wanted to see the overall type of cytokine mRNA response that was mobilized and prevailed in the tumor microenvironment as such, regardless of the cell types contributing to the establishment of the response. Our finding of significant upregulation of TNF $\beta / L T A$, a tumor necrosis factor specifically produced and secreted by activated $\mathrm{T}$ lymphocytes, reveals activated immune cells as one of the contributing cells. The up-regulation of the inflammatory cytokine trio of IL1 , TNF- $\alpha$ and particularly IL- 6 , plays a central role in the evolution of EOC. The mechanisms, by which these cytokines influence the disease progression and outcome, are complex and multifactorial [43]. Importantly, it was shown that IL-1, TNF- $\alpha$ and IL- 6 are responsible for development of symptoms, characteristic for cancer like anorexia, anemia, altered energy metabolism, weight loss, depression and fatigue and non-responsiveness to chemotherapy [43,45-47]. Elevated IL-6 levels, present in the EOC serum and malignant ascites, were associated to residual disease after debulking and to disease recurrence $[48,49]$. Furthermore, elevated IL- 6 levels were found to impair membranal IL-2 receptor expression and immune cell responses [50] and play a central role in cancer-related anemia [51,52]. TNF- $\alpha$ was shown to have direct effect on tumor cell spreading and neovascularization [53]. Thus, mounting an inflammatory response promotes several 
Citation: Israelsson P, Labani-Motlagh A, Nagaev I, Dehlin E, Nagaeva O, et al. (2017) Assessment of Cytokine mRNA Expression Profiles in Tumor Microenvironment and Peripheral Blood Mononuclear Cells of Patients with High-grade Serous Carcinoma of the Ovary. J Cancer Sci Ther 9: 422-429. doi: 10.4172/1948-5956.1000453

unwanted effects in HGSC such as stimulation of cancer-cell growth and angiogenesis, disease progression and poor disease outcome. Recently, promising attempts to counteract the negative influence of the inflammatory micromilieu in epithelial ovarian cancer by targeting TNF- $\alpha$ and IL- 6 were undertaken with promising results. Inhibition of inflammatory cytokines seemed to restore sensitivity to chemotherapy, counteract angiogenesis, reverse systemic cancer symptoms and improve survival prognosis [54-56].

In this study, we chose to measure cytokine mRNA expression levels using RT-qPCR in lieu of measuring biologically active cytokines at the protein level. The primary reason for that was that there was a time lag from collection to processing and freezing serum/plasma samples and the cytokines are sensitive to degradation in rt. Thus, we would not know the true concentration of cytokines analyzed at the protein level. The high specificity, sensitivity and stability of RT-qPCR as a method and our own long term experience and access to a well-established RTqPCR assay for simultaneous measurement of mRNA expression for a large set of cytokines was another reason to choose mRNA expression analysis $[19,20]$. Most important, our choice is supported by a previous study that showed an excellent correlation between the levels of cytokine mRNA measured by this method and measurements of corresponding cytokines at the protein level [57]. Further, RT-qPCR of tumor samples was chosen over immunohistochemical protein staining of tumor tissue since the latter is less sensitive and the semi-quantification can be easily biased. Cytokines are dynamically expressed molecules and the composition of cytokines can vary in the different ovarian cancer subtypes, between patients with the same cancer subtype, stage and grade and also in time. To minimize these expected variations between samples we have concentrated on the most malignant type of ovarian cancer, HGSC/serous EOC type II of similar grade and from the quality of the obtained results and the acceptable standard deviations we feel confident to discuss the cytokine expression in term of cytokine profiles. Furthermore, the comparability of the analyses between the samples is highlighted by the uniform and stable expression of the endogenous control that also ensured reliable quantification.

A major strength of this investigation is that in addition to comparison of HGSC patients with normal controls, patients suffering of benign ovarian conditions were included. Furthermore, simultaneous analyses were done with paired tissue- and corresponding PBMC samples thus opening a possibility to compare the cytokine mRNA expression at the local and systemic level. Another strength is the broad panel of cytokines investigated chosen to cover Th cytokine profiles of major interest. The real-time RT-qPCR analyses of mRNA for all 12 chosen cytokines was done at the same occasion to avoid experimental bias. The study groups were small with heterogeneous diagnoses for the group of benign ovarian conditions. The limited number of participants was due to difficulties to find paired blood and tissue samples from benign ovarian conditions and samples from normal ovaries. For the ovarian cancer group we selected HGSC to achieve a more homogeneous group regarding cancer subtype, grade and stage. The number of patients in the HGSC group was also reduced due to the need to exclude patients that received preoperative treatment with cytostatic drugs.

In summary, four major co-operating cytokine mRNA profiles responsible for tumour immune escape at the local and systemic level were found in HGSC patients, i.e. a profound inflammatory- and Treg immunosuppressive cytokine responses, a dysregulated local Th1 response and a Th2-deviated humoral systemic response. Future studies using larger cohorts are needed to elucidate in detail the role of cytokines in the establishment and disease progression of HGSC.
Our study contributes to evaluation of potential cytokine candidates for diagnostic cytokine mRNA profiles and therapeutic intervention by cytokine inhibition.

\section{Acknowledgments}

We are grateful to all donors, the staff at the Department of Obstetrics and Gynecology and the operating theatre, Umeå University Hospital for collecting ovarian tissue and blood samples. The Department of Pathology, Medical Biosciences is gratefully acknowledged for diagnosis and help to retrieve samples from the Ovarian Cancer Biobank at Umeå University Hospital. This work was supported by grants from the Swedish National Research Foundation (VR K201354X-22341-01-05), Cancerfonden (15 0832), Central ALF Funding, the Cancer Research foundation in Northen Sweden (www.cancerforskningsfonden.se), Lion's Cancer Research Foundation and Umeå Universitets Insamlingsstiftelse.

\section{References}

1. Lavoué V, Thédrez A, Levêque J, Foucher F, Henno S, et al. (2013) Immunity of human epithelial ovarian carcinoma: the paradigm of immune suppression in cancer. J Transl Med 11: 147

2. Gubbels JA, Claussen N, Kapur AK, Connor JP, Patankar MS (2010) The detection, treatment, and biology of epithelial ovarian cancer. J Ovarian Res 3: 8 .

3. Yigit R, Massuger LF, Figdor CG, Torensma R (2010) Ovarian cancer creates a suppressive microenvironment to escape immune elimination. Gynecol Oncol 117: 366-372.

4. Thibault B, Castells M, Delord JP Couderc B (2014) Ovarian cancer microenvironment: Implications for cancer dissemination and chemoresistance acquisition. Cancer Metastasis Rev 33: 17-39.

5. Ferlay J, Soerjomataram I, Dikshit R, Eser S (2015) Cancer incidence and mortality worldwide: Sources, methods and major patterns in GLOBOCAN. Int J Cancer 136: E359-E386.

6. Prat J; Oncology FCoG (2014) Staging classification for cancer of the ovary, fallopian tube, and peritoneum. Int J Gynaecol Obstet 124: 1-5.

7. Shih IM, Kurman RJ (2004) Ovarian tumorigenesis: A proposed model based on morphological and molecular genetic analysis. Am J Pathol 164: 1511-1518.

8. Nash MA, Ferrandina G, Gordinier M, Loercher A, Freedman RS (1999) The role of cytokines in both the normal and malignant ovary. Endocr Relat Cancer 6: 93-107.

9. Lambeck AJ, Crijns AP, Leffers N, Sluiter WJ, ten Hoor KA et al. (2007) Serum cytokine profiling as a diagnostic and prognostic tool in ovarian cancer: A potential role for interleukin 7. Clin Cancer Res 13: 2385-2391.

10. Chen LL, Ye F, Lü WG, Yu Y, Chen HZ, et al. (2009) Evaluation of immune inhibitory cytokine profiles in epithelial ovarian carcinoma. J Obstet Gynaecol Res 35: 212-218.

11. Nowak M, Glowacka E, Szpakowski M, Szyllo K, Malinowski A, et al. (2010) Proinflammatory and immunosuppressive serum, ascites and cyst fluid cytokines in patients with early and advanced ovarian cancer and benign ovarian tumors. Neuro Endocrinol Lett 31: 375-383.

12. Gorelik E, Landsittel DP, Marrangoni AM, Modugno F, Velikokhatnaya L, et al. (2005) Multiplexed immunobead-based cytokine profiling for early detection of ovarian cancer. Cancer Epidemiol Biomarkers Prev 14: 981-987.

13. Hao CJ, Li J, Liu P, Li XL, Hu YQ, et al. (2016) Effects of the balance between type 1 and type $2 \mathrm{~T}$ helper cells on ovarian cancer. Genet Mol Res 15(2).

14. Pisa P, Halapi E, Pisa EK, Gerdin E, Hising C, et al. (1992) Selective expression of interleukin 10 , interferon gamma, and granulocyte-macrophage colony-stimulating factor in ovarian cancer biopsies. Proc Natl Acad Sci U S A 89: $7708-7712$

15. Burke F, Relf M, Negus R, Balkwill F (1996) A cytokine profile of normal and malignant ovary. Cytokine 8: 578-585

16. Merogi AJ, Marrogi AJ, Ramesh R, Robinson WR, Fermin CD, et al. (1997) Tumor-host interaction: Analysis of cytokines, growth factors, and tumorinfiltrating lymphocytes in ovarian carcinomas. Hum Pathol 28: 321-331.

17. Naylor MS, Stamp GW, Foulkes WD, Eccles D, Balkwill FR (1993) Tumor necrosis factor and its receptors in human ovarian cancer. Potential role in disease progression. J Clin Invest 91: 2194-206. 
Citation: Israelsson P, Labani-Motlagh A, Nagaev I, Dehlin E, Nagaeva O, et al. (2017) Assessment of Cytokine mRNA Expression Profiles in Tumor Microenvironment and Peripheral Blood Mononuclear Cells of Patients with High-grade Serous Carcinoma of the Ovary. J Cancer Sci Ther 9: 422-429. doi: 10.4172/1948-5956.1000453

18. Tavassoli FA DP (2003) World health organization classification of tumors. Pathology and genetics of tumors of the breast and female genital organs. Lyon, France: IARC Press.

19. Nagaeva O, Jonsson L, Mincheva-Nilsson L (2002) Dominant IL-10 and TGF-beta mRNA expression in gammadelta I cells of human early pregnancy decisua suggests immunoregulatory potential. Am J Reprod Immunol 48: 9-17.

20. Winnberg A, Nagaeva O, Nagaev I, Lundell C, Arencibia I, et al. (2016) Dynamics of cytokine mRNA expression and fecal biomarkers in school children undergoing a double-blind placebo-controlled food challenge series. Cytokine 88: 259-266.

21. Bustin SA, Benes V, Carson JA, Hellemans J, Hugget J, et al. (2011) Primer sequence disclosure: A clarification of the MIQe guidelines. Clin Chem 57: 919-921.

22. National treatment and care program for ovarian cancer (2015) http:// www.cancercentrum.se/samverkan/cancerdiagnoser/gynekologi/aggstock/ vardprogram/: Regionalt cancercentrum i samverkan.

23. Sato E, Olson SH, Ahn J, Bundy B, Nishikawa H, et al. (2005) Intraepithelial CD8+ tumor-infiltrating lymphocytes and a high CD8+/regulatory T-cell ratio are associated with favorable prognosis in ovarian cancer. Proc Natl Acad Sci USA 102: 18538-18543.

24. Garzetti GG, Cignitti M, CiavattiniA, Fabris N (1993) Natural killer cell activity and progression-free survival in ovarian cancer. Gynecol Obstet Invest 35: 118-120.

25. Thedrez A, Lavoué V, Dessarthe B, Daniel P, Henna S, et al. (2013) A quantitative deficiency in peripheral blood $\mathrm{Vg} 9 \mathrm{Vd} 2$ cells is a negative prognostic biomarker in ovarian cancer patients. PLoS One 8: e63322.

26. Curiel TJ, Coukos G, Zou L, Alvarez X, Cheng P, et al. (2004) Specific recruitment of regulatory $T$-cells in ovarian carcinoma fosters immune privilege and predicts reduced survival. Nat Med 10: 942-949.

27. Strengell M, Matikainen S, Sirén J, Lehtonen A, Foster D, et al. (2003) IL-21 synergy with IL-15 or IL-18 enhances IFN-gamma production in human NK and T cells. J Immunol 170: 5464-5469.

28. Levine AG, Arvey A, Jin W, Rudensky AY (2014) Continuous requirement for the TCR in regulatory T cell function. Nat Immunol 15: 1070-1078.

29. Oleinika K, Nobbs RJ, Graham GJ, Fraser AR (2013) Suppression, subversion and escape: The role of regulatory $T$ cells in cancer progression. Clin Exp Immunol 171: 36-45

30. Krockenberger M, Dombrovski Y, Weidler C, Ossadnik M, Hönig A, et al. (2008) Macrophage migration inhibitory factor contributes to the immune escape of ovarian cancer by downregulating NKG2D. J Immunol 180: 7338-7348.

31. Ostrand-Rosenberg S, Sinha P, Beury DW, Clements VK (2012) Crosstalk between myeloid-derived suppressor cells (MDSC), macrophages, and dendritic cells enhances tumor-induced immune suppression. Semin Cancer Biol 22: 275-281.

32. Scarlett UK, Rutkowski MR, Rauwerdink AM, Fields J, Escovar-Fadul X, et al. (2012) Ovarian cancer prognosis is controlled by phenotypic changes in dendritic cells. J Exp Med 209: 495-506.

33. Liu VC, Wong LY, Jang T, Shah AH, Park I, et al. (2007) Tumor evasion of the immune system by converting CD4+CD25-T cells into CD4+CD25+ T regulatory cells: Role of tumor-derived TGF-beta. J Immunol 178: 2883-2892.

34. Conrad C, Gregorio J, Wang YH, Ito T, Meller S, et al. (2012) Plasmacytoid dendritic cells promote immunosuppression in ovarian cancer via ICOS costimulation of Foxp3(+) T-regulatory cells. Cancer Res 72: 5240-5249.

35. Vallentin B, Barlogis V, Piperouglu C, Cypowyj S, Zucchini N, et al (2017) Innate Lymphoid cells in cancer. Cancer Immunol Res CIR-15-0222.

36. Antignano F, Braam M, Hughes MR, Chenery AL, Burrows K, et al. (2016) G9a regulates group 2 innate lymphoid cell development by repressing the group 3 innate lymphoid cell program. J Exp Med 2013: 1153-1162.

37. Antignano F, Burrows K, Hughes MR, Han JM, Kron KJ, et al. (2014)
Methyltransferase G9a regulates T cell differentiation during murine intestinal inflammation. J Clin Invest 124: 1945-1955.

38. Tachibana M, Suigmoto K, Nozaki M, Ueda J, Ohta T, et al. (2002) G9a histone methyltransferase plays a dominant role in euchromatic histon $\mathrm{H} 3$ lysine 9 methylation and is essential for early embryogenesis. Genes Dev 16: 1779-1791.

39. Zhang T, Termanis A, Özkan B, Bao XX, Culley J, et al. (2016) G9a/GLP comples maintains imprinted DNA methylation in embryonic stem cells. Cell Rep 15: 77-85

40. Mantovani A, Allavena P, Sica A, Balkwill F (2008) Cancer-related inflammation. Nature 454: 436-444.

41. Sheu BC, Chang WC, Cheng CY, Lin HH, Chang DY, et al. (2008) Cytokine regulation networks in the cancer microenvironment. Front Biosci 13: 6255 6268.

42. Macciò A, Madeddu C (2012) Inflammation and ovarian cancer. Cytokine 58 : 133-147.

43. Kulbe H, Chakravarty P, Leinster AD, Charles KA, Kwong J, et al. (2011) A dynamic inflammatory cytokine network in the human ovarian cancer microenvironment. Cancer Res 72: 66-75.

44. Bennani-Baiti N, Davis MP (2008) Cytokines and cancer anorexia cachexia syndrome. Am Hosp Palliat Care 25: 407-411.

45. Argilés JM, López-Soriano FJ (1999) The role of cytokines in cancer cachexia Med Res Rev 19: 223-248.

46. Macciò A, Madeddu C, Massa D, Astara G, Farci D, et al. (2009) Interleukin-6 and leptin as markers of energy metabolic changes in advance ovarian cancer patients. J Cell Mol Med 13: 3951-3959.

47. Scambia G, Testa U, Benedetti Panici P, Foti E, Martucci R, et al. (1995) Prognostic significance of interleukin- 6 serum levels in patients with ovarian cancer. Br J Cancer 71: 354-356.

48. Plante M, Rubin SC, Wong GY, Federici MG, Finstad CL, et al. (1994) Interleukin-6 level in serum an ascites as a prognostic factor in patients with epithelial ovarian cancer. Cancer 73: 1882-1888.

49. Macciò A, Lai P, Santona MC, Pagliara L, Melis GB, et al. (1998) High serum levels of soluble IL-2 receptor, cytokines and C-reactive protein correlate with impairment of $T$ cell response in patients with advanced epithelial ovarian cancer. Gynecol Oncol 69: 248-252.

50. van der Zee AG, de Cuyper EM, Limburg PC, de Bruij HW, Hollema $\mathrm{H}$, et al. (1995) High levels of interleukin-6 in cystic fluids from patients with malignant versus benign ovarian tumors correlate with decreased hemoglobin levels and increased platelet counts. Cancer 75: 1004-1009.

51. Macciò A, Madeddu C, Massa D, Mudu MC, Lusso MR, et al. (2005) Hemoglobin levels correlate with interleukin-6 levels in patients with advanced untreated epithelial ovarian cancer: Role of inflammation in cancer-related anemia. Blood 106: 362-367.

52. Kulbe H, Thompson R, Wilsson JL, Robinson S, Hagemann T, et al. (2007) The inflammatory cytokine tumor necrosis factor-alpha generates an autocrine tumor-promoting network in epithelial ovarian cancer cells. Cancer Res 67 585-592.

53. Guo Y, Nemeth J, O'Brian C, Susa M, Liu X, et al. (2010) Effects of Siltuximab on the IL-6-induced signalling pathway in ovarian cancer. Clin Cancer Res 16 : 5759-5769.

54. Coward G, Kulbe H, Chakravarty P, Leader D, Vassileva V, et al. (2011) Ilnterleukin-6 as therapeutic agent in human ovarian cancer. Clin Cancer Res 17: $6083-6096$

55. Gavalas N, Karadimou A, Dimopoulos MA, Bamias A (2010) Immune response in ovarian cancer: How is the immune system involved in prognosis and therapy. Clin and Dev Immunol.

56. West CE, Hernell O, Andersson Y, Sjöstedt M, Hammarström ML (2012) Probiotic effects on T-cell maturation in infants during weaning. Clin Exp Allergy 42: $540-549$ 